\title{
Incidence of postoperative epilepsy after a transtentorial approach to acoustic nerve tumours
}

\author{
R. CA B R A L ${ }^{1}, T . T . K I N G, A N D$ D. F. S C O T T \\ From the Section of Neurological Sciences, The London Hospital, London
}

SYNOPS IS Sixty-nine patients who had neurosurgical treatment for acoustic neuroma by one of two different techniques were studied with a view to determining the incidence of postoperative epilepsy. Forty-five patients who had larger tumours underwent a combined translabyrinthine and transtentorial neurosurgical approach. For the others with smaller neuromas a translabyrinthine method was used. Only the combined approach was associated with postoperative epilepsy, and it occurred in $22 \%$ of the patients. Epilepsy was associated with temporal lobe trauma during surgery.

Removal of an acoustic tumour, whatever the technique employed, presents operative risks, both to the life of the patient and useful survival (Northfield, 1973). Though epilepsy has not been reported as a consequence of removing an acoustic neuroma by the posterior fossa approach (MacKenzie, 1965), in a series of 23 large or medium sized tumours excised by the combined translabyrinthine and transtentorial technique $13 \%$ of the patients developed epilepsy (Morrison and King, 1973). One of the main aims of the present investigation was to compare the incidence and evolution of postoperative epilepsy after two neurosurgical approaches, firstly the translabyrinthine procedure in which the approach is through the internal auditory canal and the posterior fossa, and secondly the combined translabyrinthine and transtentorial technique (King, 1970), in which the temporal lobe is exposed and may suffer operative trauma. Further, an attempt was made to assess the type of interoperative and postoperative complications which might be related to the development of epilepsy.

\footnotetext{
${ }^{1}$ This study formed part of R.C.'s PhD Thesis accepted by the University of London.

(Accepted 27 February 1976.)
}

\section{METHODS}

There were 69 patients who underwent surgery for acoustic neuroma at the London Hospital between 1968 and 1974 and who had been followed up postoperatively for at least a year (average 41 months with a range of 12 to 88 months). Thirty-two patients were male and 37 female. The mean age for the whole group was 46 years, ranging from 19 to 67 years. Thirty-two of the 69 patients had large acoustic neuromas, that is more than $3 \mathrm{~cm}$ in diameter; 31 had medium sized tumours measuring between 1 and $3 \mathrm{~cm}$, and in the remaining six the tumour was even smaller and confined to the internal auditory canal.

All patients with a large acoustic neuroma underwent the combined translabyrinthine, transtentorial approach to the tumour, a technique (Morrison and King, 1973) which involves the retraction of the exposed temporal lobe and the division of the tentorium to gain access to the cerebello-pontine angle. Thirteen patients with a medium sized tumour also had this operation. The translabyrinthine technique was used in 24 patients, 18 of whom had a medium sized tumour. The mean ages and sex distribution were similar in the two surgical groups. In order to determine what factors were related to the occurrence of epilepsy special attention was paid to untoward events during surgery-for example, swelling of the temporal lobe, bleeding from veins, brain stem or cerebellar trauma. Similarly, postopera- 
tive sequelae such as dysphasia and depression of consciousness werc also studied. These features were assessed according to two categories, either minimal or severe. Subsequently, patients who sustained definite intraoperative or postoperative sequelac were contrasted with those in whom negligible complications had occurred. The association between epilepsy and these various types of complication was calculated by means of the chi-square test.

\section{RESULTS}

Ten patients $(14 \%)$ of the total 69 cases developed postoperative epilepsy. All these had undergone the combined neurosurgical approach and thus the incidence in that group was $22 \%$, a statistically significant difference $(\mathrm{P}<0.05)$, when compared with the translabyrinthine approach. The mean latent period between surgery and the occurrence of the first seizure was 10 months, ranging from the first week up to four years (Table). Eight patients had their initial seizure in the first year after operation, while in four 'early' epilepsy occurred-that is, their first seizure was during the first postoperative week. Two patients had status epilepticus during that period and another within the first month of operation. This followed protracted meningitis and hydrocephalus and the patient died 18 months later. Four patients had generalized seizures and two had partial seizures, one with psychomotor symptomatology. Four further patients had partial seizures which became secondarily generalized.

In spite of anticonvulsants seven patients continued to have seizures. However, of thesc onc patient had fits as frequently as onc per month, a further patient had one seizure per three months, and the majority had only one attack per year. Seizures did not have any marked social effect in seven patients and they were able to return to their employment. Two had difficulties of adjustment after the development of fits, one other being the patient who died having been physically disabled since operation.

The relationship of surgical and postoperative complications to epilepsy was studied. In the group of 45 patients who under- went the combined approach to the-tumour there was an association between intraoperative trauma to the temporal lobe and epilepsy $(\mathrm{P}<0.01)$ Conversely, it was found that negligible or mild complications during surgery were not related to the occurrence of cpilepsy $(\mathrm{P}<0.01)$. There was a tendency, which did not reach significance, for patients with left-sided acoustic neuromas to develop seizures. These occurred in eight out of 10 patients who had tumours on that side. Moderate or severe postoperative signs and symptoms, particularly dysphasia, correlated significantly with development of epilepsy $(\mathrm{P}<0.02)$.

\section{T A B L E}

LATENT PERIOD IN MONTHS, FOR DEVELOPMENT OF EPILEPSY IN 10 PATIENTS WHO UNDERWENT SURGERY FOR ACOUSTIC NEUROMA (MEAN 10 MONTHS)

\begin{tabular}{lllllllll}
\hline & & \multicolumn{7}{c}{ Months } \\
& 1 & 2 & 3 & 4 & 12 & 37 & 48 \\
\hline Number of patients & 4 & 1 & 1 & 1 & 1 & 1 & 1
\end{tabular}

\section{DISCUSSION}

The combined translabyrinthine, transtentorial approach to acoustic neuroma has been of value as mortality is low. However, this study indicates that morbidity may occur in the form of postoperative seizures. The incidence is somewhat greater than the previous figure (Morrison and King, 1973) and this may well be related to the greater length of follow-up in the present study. It appears that seizures, however, are on the whole treatable with drugs and tend not to lead to poor social adjustment. Though the latent period between the development of seizures after surgery varies widely, it is of interest that three out of the four patients who had 'early' epilepsy also had subsequent fits. These findings are therefore somewhat at variance with suggestions-for example, in the case of head injury - that there is little correspondencc between 'early' and 'late' epilepsy (Penfield and Jasper, 1954) and are in line with the recent report of Jennett (1974). There 
was also an interesting association between left-sided tumours and the occurrence of epilepsy similar to that observed by McClelland, Annett, and Scott (1974, unpublished) in patients with cerebral abscess.

The size of the tumour seemed to bear an important relationship to the occurrence of epilepsy, as only those with large acoustic neuromas presented postoperative seizures. This may be because the length of the operation in these patients is greater, entailing a more prolonged retraction of the temporal lobe. Certainly the group of patients who developed seizures presented a significantly higher proportion of temporal lobe damage and postoperative dysphasia than did the cases without epilepsy. Presumably this was related to temporal lobe injury leading to oedema, hypoxia, and subsequently scarring. Another indication of the probable epileptogenic nature of the temporal lesion is the fact that fits have been reported (Jannetta, 1974) after a microsurgical transtentorial approach to the trigeminal nerve, a procedure which also requires retraction of the temporal lobe.

Epilepsy emerged in this study as an important complication of the transtentorial approach to the cerebello-pontine angle tumour. For this reason, two courses of action have been pursued. Firstly, the technique has been modified so that retraction of the temporal lobe is more restricted. Secondly, anticonvul- sants have been administered routinely to these patients immediately postoperatively, and continued for 18 months. These measures together seem to have reduced the incidence of epilepsy.

We acknowledge the help of $\mathrm{Mr}$ Andrew Morrison, senior ENT surgeon at the London Hospital, whose interest in acoustic neuroma surgery has provided the material for this study.

\section{REFERENCES}

Jannetta, P. J. (1974). Complications from microsurgical treatment of tic douloureux. Journal of Neurosurgery, 40, 675.

Jennett, B. (1970). Early traumatic epilepsy: incidence and significance after non-missile injuries. Archives of Neurology (Chic.), 30, 394-398.

King, T. T. (1970). Combined trans-labyrinthine and trans-tentorial approach to acoustic nerve tumours. Proceedings of the Royal Society of Medicine, 63, $780-782$.

MacKenzie, I. (1965). Consequences of removing an acoustic neuroma by conventional surgical methods. Proceedings of the Royal Society of Medicine, 58, 1071.

Morrison, A. W., and King, T. T. (1973). Experiences with a trans-labyrinthine trans-tentorial approach to the cerebello-pontine angle: technical note. Journal of Neurosurgery, 38, 382-390.

Northfield, D. W. C. (1973). The Surgery of the Central Nervous System. Blackwell: Oxford.

Penfield, W., and Jasper, H. H. (1954). Epilepsy and the Functional Anatomy of the Human Brain. Little, Brown: Boston. 